\title{
The Physical Presence of Vitamin E in Lipid Membranes
}

\author{
Mitchell DiPasquale, Michael H.L. Nguyen, Brett W. Rickeard, and Drew Marquardt \\ Department of Chemistry and Biochemistry, University of Windsor, Windsor, ON, Canada
}

Since its discovery over 90 years ago, the biological significance of vitamin E (tocopherol) has been surrounded by controversy. 1,2 Physiologically, Vitamin E deficiency has been correlated to several health disorders yet clear molecular mechanisms for many these phenotypes remain elusive. ${ }^{3,4}$ Numerous studies have found $\alpha$-tocopherol, the predominant species of vitamin E, to be an antioxidant in vitro, often interpreting the biomolecules function to be fat-soluble peroxyl radical scavenger, ${ }^{5}$ however, others have directly opposed this theory. ${ }^{6}$ The antioxidant theory is supported by studies by Marquardt et al, in which neutron diffraction reveals the transverse membrane orientation of $\alpha$-tocopherol to be appropriate for interception of reactive oxygen species as well as to terminate lipid-borne radicals. ${ }^{7}$ Studies have since diverged to show that $\alpha$-tocopherol may in fact have vital roles in cellular processes such as signaling, apoptosis, protein activity, and gene regulation. $4,6,8$ Considering the lack of conclusive investigations, the absence of a clear antioxidant health benefit from supplementation, and its significantly low physiological concentrations, skepticism builds regarding the true biological function of vitamin E. It is known to localize in lipid membranes, and so investigations on the physical effect produced by $\alpha$ - tocopherol are vital, yet absent.

Preliminary experiments employing gas chromatography and densitometry suggested that $\alpha$-tocopherol favours localization in less ordered environments, such as those provided by unsaturated lipids. In the context of biological membrane systems, cholesterol is vital in the maintenance of membrane integrity by promoting lipid organization into phases of liquid-ordered (Lo) and liquiddisordered $(\mathrm{Ld})$ domains, which allow for the formation of functional lipid rafts. ${ }^{9}$ The latter of these phases is favourable for tocopherol localization.

The physical perturbation of the lipid bilayer induced by various species of tocopherol ( $\alpha$-tocopherol, $\gamma$-tocopherol, $\alpha$ tocopherylquinone) was observed through small angle neutron scattering coupled with contrast matching techniques. ${ }^{10,11}$ Symmetric unilamellar vesicles of an established domain-forming lipid composition were prepared and doped with a species of tocopherol. ${ }^{12}$ A decrease in scattering intensity at a q-range $\left(0.004-0.04 \AA^{-1}\right)$ corresponding to domain size is indicative of a smaller area fraction of the membrane system being present in the ordered phase. This variance in scattering intensity is quantified with the Porod invariant to compare the abundance of Lo domains, as well as the stability of these domains as they melt dependent on temperature. Results indicate that increasing tocopherol concentrations correlates to both the reduction and destabilization of ordered domains. This effect is evident with $\alpha$-tocopherol and $\gamma$-tocopherol and is much less pronounced in the quinone-doped system.

Alterations in the membranes physical properties justifies the significance of vitamin $\mathrm{E}$ in membrane systems, representing a biological role beyond that of purely an antioxidant.

1. Brigelius-Flohé, R. \& Davies, K. J. A. Is vitamin E an antioxidant, a regulator of signal transduction and gene expression, or a 'junk' food? Comments on the two accompanying papers: 'Molecular mechanism of $\alpha$-tocopherol action' by A. Azzi and 'Vitamin E, antioxidant and nothing more' by M. Tra. Free Radic. Biol. Med. 43, 2-3 (2007).

2. Brigelius-Flohé, R. \& Galli, F. Vitamin E: A vitamin still awaiting the detection of its biological function. Mol. Nutr. Food Res. 54, 583-587 (2010).

3. Evans, H. \& Bishop, K. On the existence of a hitherto unrecognized dietary factor essential for reproduction. Science (80). 56, 650-651 (1922).

4. Gohil, K., Vasu, V. T. \& Cross, C. E. Dietary $\alpha$-tocopherol and neuromuscular health: Search for optimal dose and molecular mechanisms continues! Mol. Nutr. Food Res. 54, 693-709 (2010).

5. Buettner, G. R. The Pecking Order of Free Radicals and Antioxidants: Lipid Peroxidation, alpha- Tocopherol and Ascorbate. Arch. Biochem. Biophys. 300, 535-543 (1993).

6. Azzi, A. Molecular mechanism of $\alpha$-tocopherol action. Free Radic. Biol. Med. 43, 16-21 (2007).

7. Marquardt, D., Kucerka, N., Katsaras, J. \& Harroun, T. A. $\alpha$ - Tocopherol's Location in Membranes Is Not Affected by Their Composition. Langmuir 31, 4464-4472 (2015).

8. Noguchi, N., Hanyu, R., Nonaka, A., Okimoto, Y. \& Kodama, T. Inhibition of THP-1 Cell Adhesion to Endothelial Cells by $\alpha$-tocopherol and $\alpha$-tocotrienol is Dependent on Intracellular Concentration of the Antioxidants. Free Radic. Biol. Med. 34, 1614-1620 (2003).

9. Yeagle, P. L. Non-covalent binding of membrane lipids to membrane proteins. Biochim. Biophys. Acta - Biomembr. 1838, 1548-1559 (2014).

10. Pencer, J. et al. Detection of submicron-sized raft-like domains in membranes by small- angle neutron scattering. Eur. Phys. J. E 18, 447-458 (2005).

11. Pan, J., Heberle, F. A., Petruzielo, R. S. \& Katsaras, J. Using small-angle neutron scattering to detect nanoscopic lipid domains. Chem. Phys. Lipids 170-171, 19-32 (2013).

12. Veatch, S.L. \& Keller, S.L. Separation of Liquid Phases in Giant Vesicles of Ternary Mixtures of Phospholipids and Cholesterol. Biophys. J. 85, 3074-3083 (2003).

Acta Cryst. (2018). A74, a152 